\title{
PREFERENCIA DE CUATRO ESPECIES DE PLANTAS AL DRENAJE Y LA ALTURA EN LOS BOSQUES SOBRE ARENA BLANCA EN LA ZONA RESERVADA ALLPAHUAYO - MISHANA
}

\author{
Manuel A. Ahuite Reáteguii ${ }^{1}$, Roosevelt García Villacorta ${ }^{1}$ y Kalle Ruokolainen ${ }^{2}$
}

\author{
RESUMEN
}

Estudiamos las preferencias respecto a las condiciones de drenaje y la altura del bosque en cuatro especies de plantas restringidas a suelos de arena blanca en la Zona Reservada Allpahuayo - Mishana, Loreto, Perú. En 44 parcelas de $20 \times 20 \mathrm{~m}$ contamos el número total de tallos (plantas mayores de $1 \mathrm{~m}$ de altura) de Caraipa tereticaulis Tul. (1 032 tallos), C. utilis Vásquez (2 304 tallos), Euterpe catinga Wallace (994 tallos), y Pachira brevipes (A. Robyns) W. S. Alverson (4 988 tallos). En cada parcela medimos, además, el grosor de la capa de materia orgánica y altura del dosel con clinómetro. Usamos el grosor de la capa de materia orgánica como indicador de la calidad del drenaje en el suelo (materia orgánica gruesa = mal drenaje, y viceversa). Nuestros resultados indican que las cuatro especies prefieren alturas intermedias del bosque; sin embargo, $P$. brevipes tuvo el rango más amplio, y E. catinga estuvo más restringida a alturas intermedias. $C$. utilis prefiere lugares húmedos con mal drenaje, $C$. tereticaulis lugares húmedos y a veces lugares intermedios, $E$. catinga es bastante restringida a condiciones húmedas, y $P$. brevipes aparentemente no tiene una preferencia específica con respecto al drenaje.

Palabras clave: Altura del dosel, bosques sobre arena blanca, Caraipa tereticaulis, Caraipa utilis, drenaje, Euterpe catinga, materia orgánica, Pachira brevipes, varillales, Zona Reservada Allpahuayo - Mishana.

\begin{abstract}
We studied preferences relating to drainage conditions and forest canopy height in four species of plants restricted to soils of white-sand forest in the Allpahuayo-Mishana Reserved Zone, Loreto, Peru. In 44 plots of 20 x 20m, we counted the total number of stems (plants higher than $1 \mathrm{~m}$ ) of Caraipa tereticaulis Tul. (1 032 stems), C. utilis Vasquéz (2 304 stems), Euterpe catinga Wallace (994 stems) and Pachira brevipes (A. Robyns) W. S. Alverson (4 988 stems). In each plot we measured the thickness of organic layer and canopy height using a clinometer. We used the thickness of the organic layer as an indicator of the drainage quality of the soil (thick organic layer $=$ poor drainage and vice versa). Our results show that the four species prefer intermediate forest heights; however, $P$. brevipes has a wider range and E. catinga is more restricted to intermediate height. $C$. utilis prefers wet places with poor drainage, $C$. tereticaulis prefers wet places and sometimes intermediate places, $E$. catinga is quite restricted to wet conditions, and $P$. brevipes does not show a clear preference of drainage conditions.
\end{abstract}

Key words: Allpahuayo-Mishana Reserved Zone, canopy height, Caraipa tereticaulis, Caraipa utilis, drainage, Euterpe catinga, organic material, Pachira brevipes, white-sand forests.

1 Proyecto Diversidad Biológica de la Amazonía Peruana - BIODAMAZ, Perú - Finlandia, Av. Abelardo Quiñones km 2.5, Iquitos, Perú. Correo electrónico: biodamaz@iiap.org.pe

2 Proyecto Diversidad Biológica de la Amazonía Peruana - BIODAMAZ, Perú - Finlandia, Av. Abelardo Quiñones km 2.5, Iquitos, Perú. / Departamento de Biología, Universidad de Turku, 20014 Turku, Finlandia. Correo electrónico: biodamaz@iiap.org.pe 


\section{INTRODUCCIÓN}

Los bosques que crecen en climas tropicales lluviosos, según la mayoría de los autores, tienen una estructura bastante compleja, con árboles emergentes gigantes y varias capas de dosel, así como lianas, epifitas, y otras formas vegetales (Holdridge, 1967; Walter y Breckle, 1967). Existen varias excepciones a esta generalización, y una de ellas es la vegetación sobre arena blanca, que es generalmente más densa y baja que los demás tipos de bosques (Anderson, 1981; Encarnación, 1985). (Kalliola et al., 1993; Kalliola y Flores, 1998).

La Zona Reservada Allpahuayo - Mishana (ZRAM) ha sido creada para preservar una muestra representativa de los bosques sobre arena blanca (localmente conocidos como varillales), y la excepcional diversidad biológica del área de Iquitos. Existen varios tipos de varillales estructuralmente diferentes. Los criterios usados para definir los tipos de estos varillales son drenaje y altura del dosel. Sin embargo, no se ha documentado sistemáticamente preferencias de especies de plantas a los tipos de varillales, aunque algunas observaciones sugieren que posiblemente algunas especies prefieren ciertos tipos de bosques (Revilla, 1974; Ruokolainen y Tuomisto, 1993; 1998; Tuomisto y Ruokolainen, 1994; Álvarez y Soini, 2000).

Como parte del proyecto para la clasificación de los bosques sobre arena blanca de la ZRAM (García et al., 2002), en el marco del proyecto Diversidad Biológica de la Amazonía Peruana, Perú - Finlandia (BIODAMAZ) ${ }^{3}$, se estudió la composición florística, estructura y características ambientales de 44 parcelas ubicadas en la ZRAM. A continuación presentamos los resultados de un estudio sobre la preferencia de hábitat de cuatro especies de plantas características de estos bosques sobre arena blanca. Este informe recoge algunos datos referidos a las especies en cuestión del proyecto "Clasificación de los Varillales", financiado por el proyecto BIODAMAZ.

El presente estudio tuvo como objetivos principales estudiar la preferencia (densidad de tallos), de cuatro especies características de varillales (Caraipa utilis, C. tereticaulis, Euterpe catinga y Pachira brevipes) (Missouri Botanical Garden - $\mathrm{w}^{3}$ TROPICOS, 2002), con respecto a la altura del bosque y al drenaje (medida directamente por el grosor de la materia orgánica).

\section{MATERIAL Y MÉTODO}

\section{1. Área de estudio}

El presente estudio se realizó en la Zona Reservada Allpahuayo - Mishana. Esta Zona Reservada se ubica en la Amazonía peruana al sur oeste de la ciudad de Iquitos, provincia de Maynas, departamento de Loreto, entre el río Nanay y la carretera Iquitos - Nauta $\left(03^{\circ} 58.560^{\prime}\right.$ S, $7^{\circ} 25.231^{\prime}$ O; altitud entre 110 y 180 m.s.n.m) (Vásquez, 1993), y tiene una superficie de 57663.43 ha. La ZRAM protege una variedad de formaciones vegetales características de la región (bosques sobre arena blanca, localmente conocidos como varillales y chamizales), las que se caracterizan por presentar un alto endemismo de especies (Álvarez y Soini, 2000).

\subsection{Trabajo de campo}

Para identificar las zonas de muestreo utilizamos una imagen de satélite Landsat TM, que está disponible en el Sistema de Información de la Diversidad Biológica y Ambiental de la Amazonía Peruana (SIAMAZONIA; www.siamazonia.org.pe), aunque utilizamos una versión con mayor resolución (30 x 30 m) proporcionado por el Centro de Información Geográfica de la Amazonía Peruana (CIGAP), del Instituto de Investigaciones de la Amazonía Peruana (IIAP). En estas imágenes pudimos ver que los varillales muestran colores distintos a otros

3 Proyecto Diversidad Biológica de la Amazonía Peruana (BIODAMAZ) es un convenio entre los gobiernos del Perú y de Finlandia ejecutado conjuntamente por el Instituto de Investigaciones de la Amazonía Peruana (IIAP) y por el consorcio finlandés formado por la empresa de consultorías ambientales, Biota BD Oy, y por la Universidad de Turku. 
tipos de bosque. Además, dentro de los mismos varillales pudimos observar también variaciones de colores. Nuestras zonas de muestreo fueron localizadas con el objeto de maximizar los colores incluidos en el muestreo. De esta forma hemos muestreado una gran variedad de diferentes tipos de varillal, porque pensamos que los diferentes colores en la imagen indican las variaciones de los tipos de varillal.

Elaboramos 44 parcelas de 20 × $20 \mathrm{~m}$ cada una, siempre dentro de bosques sobre arena blanca, con el número de 16 sub-parcelas de 5 x 5 m, y dentro de éstas contamos los tallos de todos los individuos mayores de $1 \mathrm{~m}$ de altura de Caraipa utilis, C. tereticaulis, Euterpe catinga y Pachira brevipes.

También medimos con un clinómetro la altura del bosque dentro de cada parcela. Buscamos un árbol representativo del dosel, desde el cual tensábamos la wincha hasta los $15 \mathrm{~m}$ y medimos la altura del árbol, obteniendo así una medida de la altura promedio del bosque.

El grosor de la capa de materia orgánica sobre suelo mineral fue medido con una wincha de $3 \mathrm{~m}$ en un hueco practicado con un machete. La medición fue hecha en cinco huecos dentro de cada parcela (en las cuatro esquinas y en el centro), y el promedio fue usado como la medición final. Consideramos el grosor de la materia orgánica como un indicador de drenaje, en el sentido de que cuanta más gruesa era la capa orgánica, más deficiente era el drenaje.

\section{RESULTADOS}

En las Figuras 1 y 2 se puede observar la abundancia de Caraipa tereticaulis en relación con el grosor de la capa de materia orgánica del suelo y la altura promedio del dosel del bosque. Esta especie es más abundante en varillales intermedios, varillales bajos, y en chamizales (para la terminología de tipos de varillal, ver García et al., 2002). Sin embargo, está ausente en varillales altos y varillales con muy poca materia orgánica.

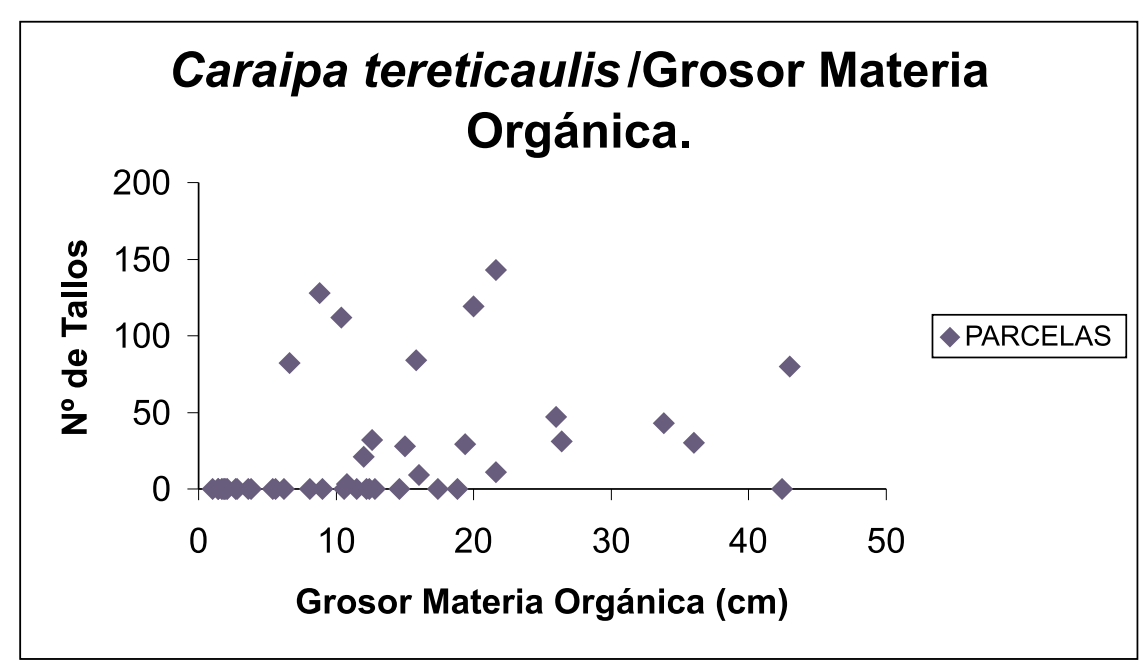

Figura 1. Representación gráfica de la abundancia de Caraipa tereticaulis con respecto al grosor de la capa de materia orgánica. Cada punto representa una parcela de muestreo. 


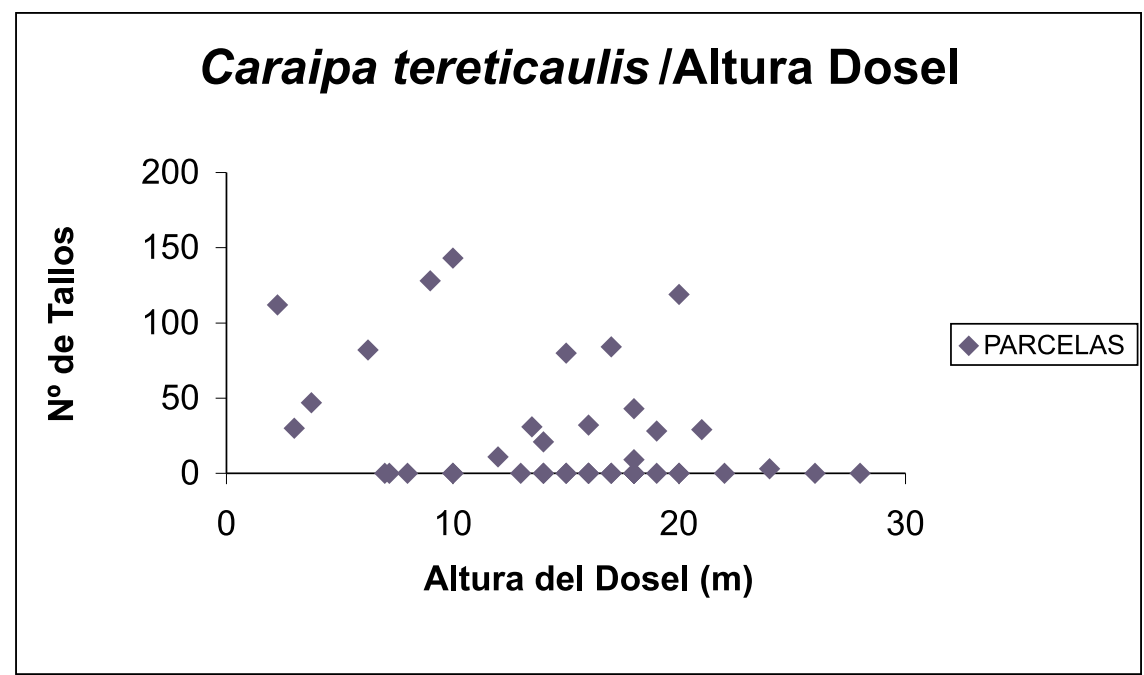

Figura 2. Representación gráfica de la abundancia de Caraipa tereticaulis con respecto a la altura del dosel. Cada punto representa una parcela de muestreo.

En las Figuras 3 y 4 se puede observar la abundancia de Caraipa utilis en relación con el grosor de la capa de materia orgánica del suelo y la altura promedio del dosel del bosque, respectivamente. Esta especie es más abundante en varillales intermedios y bajos. No se presenta en chamizales ni en varillales altos. Encontramos la especie en parcelas con grosor de materia orgánica muy variable, pero más abundante era en parcelas con grosor alrededor de 10-20 cm, aproximadamente.



Figura 3. Representación gráfica de la abundancia de Caraipa utilis con respecto al grosor de la capa de materia orgánica. Cada punto representa una parcela de muestreo. 


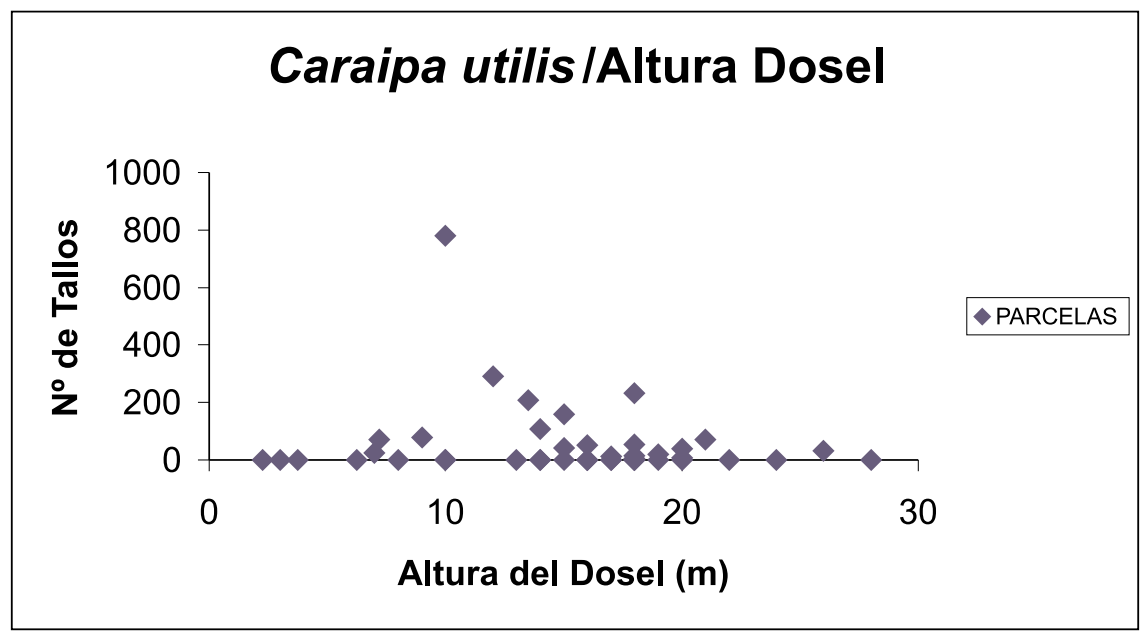

Figura 4. Representación gráfica de la abundancia de Caraipa utilis con respecto a la altura del dosel. Cada punto representa una parcela de muestreo.

En las Figuras 5 y 6 se puede observar la abundancia de Euterpe catinga en relación al grosor de la capa de materia orgánica y la altura promedio del bosque. Esta especie es más abundante en varillales intermedios. No está presente en varillales altos ni en chamizales. Encontramos la especie en parcelas con grosor muy variable en la capa de materia orgánica, pero era más abundante en parcelas cuya capa orgánica tenía un grosor de alrededor de $10-20 \mathrm{~cm}$.



Figura 5. Representación gráfica de la abundancia de Euterpe catinga con respecto al grosor de la capa de materia orgánica. Cada punto representa una parcela de muestreo. 


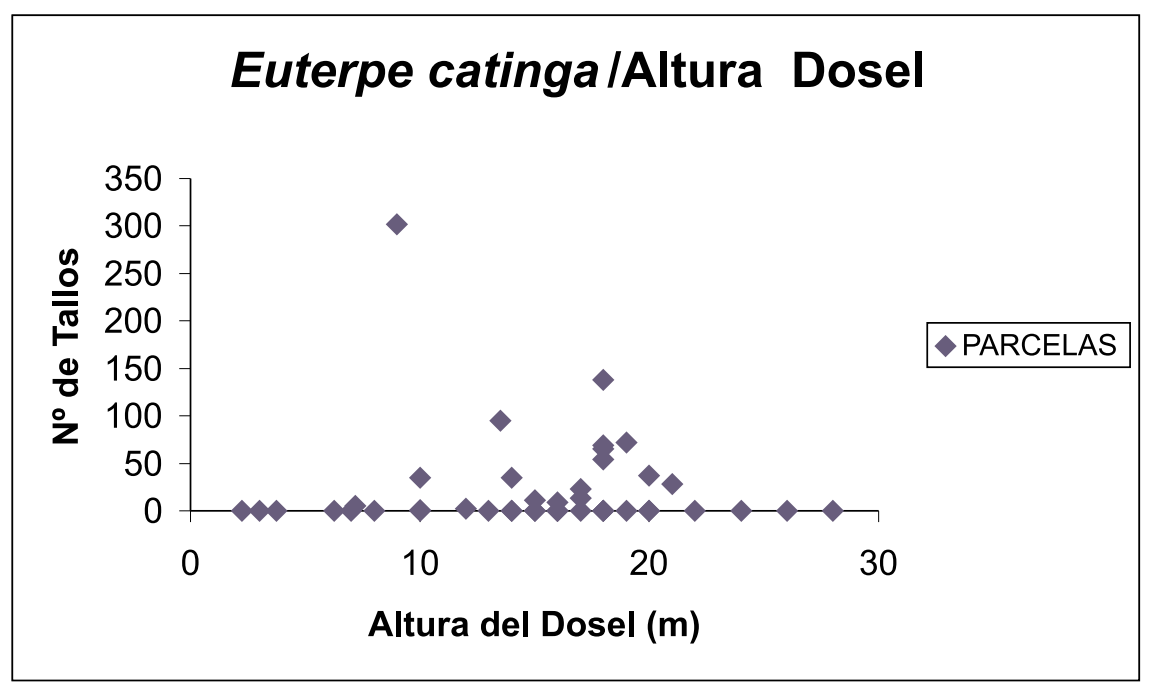

Figura 6. Representación gráfica de la abundancia de Euterpe catinga con respecto a la altura del dosel. Cada punto representa una parcela de muestreo.

En las Figuras 7 y 8 se puede observar la abundancia de Pachira brevipes en relación con el grosor de la capa de materia orgánica y la altura promedio del bosque. Esta especie está presente en todos los varillales, pero es más abundante cuando la altura del dosel está entre más o menos 8 y $20 \mathrm{~m}$.

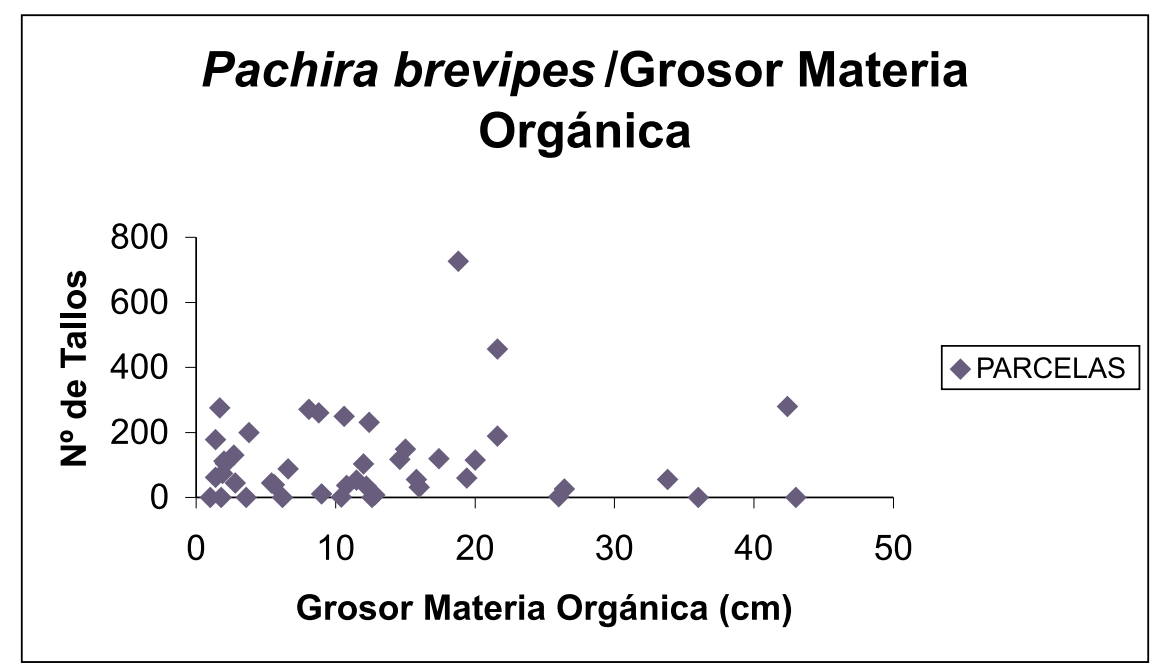

Figura 7. Representación gráfica de la abundancia de Pachira brevipes con respecto al grosor de la capa de materia orgánica. Cada punto representa una parcela de muestreo. 


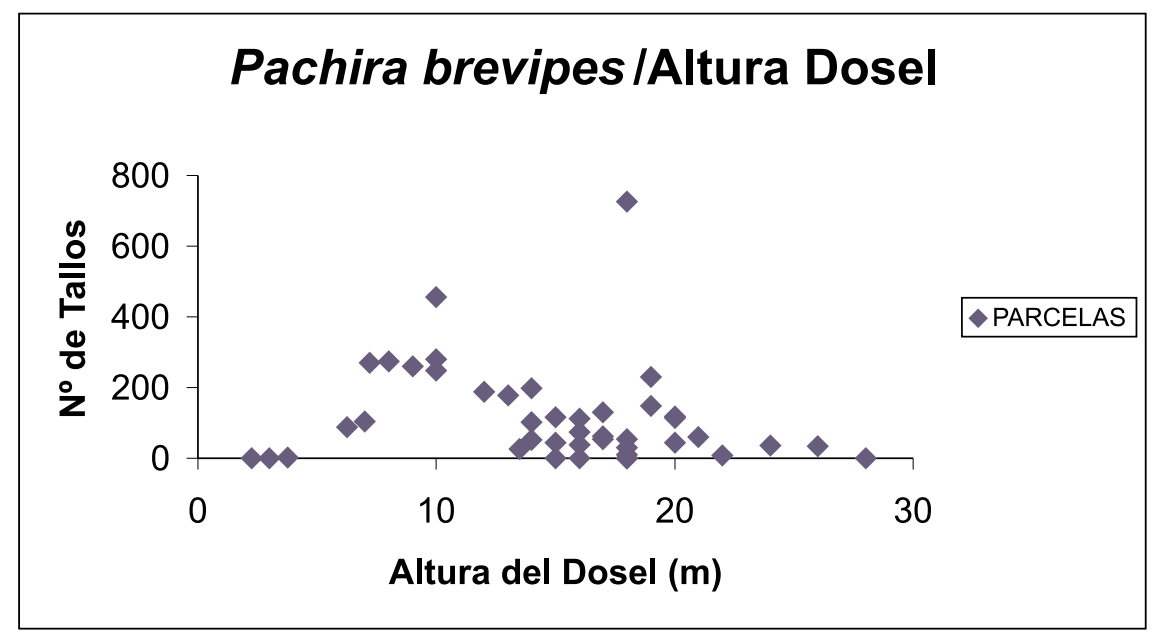

Figura 8. Representación gráfica de la abundancia de Pachira brevipes con respecto a la altura promedio del dosel. Cada punto representa una parcela de muestreo.

\section{DISCUSIÓN}

Caraipa tereticaulis prefiere lugares húmedos con mal drenaje (con regular abundancia de materia orgánica, y a veces mucha), y raras veces lugares secos con buen drenaje (poca materia orgánica). Respecto a la altura de dosel, prefiere varillales intermedios y bajos, aunque también se encuentra a esta especie en los chamizales, y más raramente en varillales altos secos.

Caraipa utilis prefiere lugares secos con buen drenaje (con poca materia orgánica) y lugares húmedos con mal drenaje (regular cantidad de materia orgánica, y raras veces con mucha). Respecto a la altura del dosel, prefiere varillales intermedios y bajos, aunque algunos individuos están presentes también en varillales altos. Sin embargo, no se encuentra en chamizales.

Estas dos especies son útiles para la industria de la madera y son usadas comúnmente por los lugareños de la ZRAM. Por tal motivo se debería realizar estudios sobre ecología, crecimiento, regeneración natural y su respuesta a la extracción, con miras a lograr un adecuado manejo y un uso sostenido de este recurso.

Euterpe catinga prefiere lugares húmedos con mal drenaje (con regular cantidad de materia orgánica y a veces con mucha) y lugares secos con buen drenaje (con poca materia orgánica). En relación con la altura del dosel, prefiere varillales intermedios, y algunos individuos están también presentes en varillales bajos. Sin embargo, la especie no está presente en chamizales ni en varillales altos secos.

Pachira brevipes aparentemente no tiene una preferencia específica con respecto al drenaje. En relación con la altura del dosel, prefiere varillales intermedios y bajos, aunque también se la encuentra con menor abundancia en varillales altos. No está presente en chamizales. Esta especie parece ser la más generalista de las especies estudiadas.

Las cuatro especies también fueron mencionadas en el trabajo preliminar realizado por Álvarez y Soini (2000). Ellos aseguran que $C$. tereticaulis y E. catinga no están presentes en varillales secos y; $C$. utilis no está presente en varillales intermedios y bajos húmedos. Nuestros resultados están más o menos de acuerdo con lo que se 
reportó de E. catinga y C. tereticaulis, pero C. utilis en nuestro estudio fue encontrado en varillales intermedios húmedos y bajos húmedos. Para aclarar estas discrepancias en los resultados es necesario realizar más estudios.

\section{CONCLUSIONES}

C. tereticaulis, $C$. utilis y E. catinga tienen preferencias por varillales húmedos con mal drenaje. La mayor diferencia entre estas tres especies es que $C$. tereticaulis es muy abundante en chamizales, en los cuales no se encuentran $C$. utilis ni E. catinga. P. brevipes parece ser una generalista en comparación con las otras tres especies.

\section{AGRADECIMIENTOS}

Nuestro agradecimiento al proyecto BIODAMAZ, Perú - Finlandia, por la oportunidad de realizar este trabajo.

\section{BIBLIOGRAFÍA}

ÁLVAREZ, J.; SOINI, P. 2000. Evaluación y Conservación de la Diversidad Biológica para el Ecoturismo. En: Informe final del sub proyecto Evaluación de la Fauna Silvestre del área de influencia la carretera Iquitos, Perú. PBIO-IIAP, Iquitos, Perú. 14-19 pp.

ANDERSON, A.B.1981. White-sand vegetation of Brazilian Amazonia. Biotropica 13:199-210.

ENCARNACIÓN, F.1985. Introducción a la Flora y Vegetación de la Amazonía peruana: estado actual de los estudios, medio natural y ensayo de claves de la determinación de las formaciones vegetales en la llanura Amazónica. Candollea 40:237-252.

GARCÍA, R.; AHUITE, M.; OLÓRTEGUI, M. 2002. Clasificación de bosques sobre arena blanca de la Zona Reservada Allpahuayo-Mishana. Folia Amazónica 14 (1-2):17-33 pp.

HOLDRIDGE, L.R. 1967. Life Zone Ecology. Tropical Science Center, San José, Costa Rica.

KALLIOLA, R.; FLORES, S. (eds.). 1998. Geoecología y desarrollo amazónico: estudio integrado en la zona de Iquitos, Perú. Annales Universitatis Turkuensis Ser. A II. 114. 544 pp.

KALLIOLA, R.; PUHAKKA, M.; DANJOY, W. (eds.). 1993. Amazonía peruana. Vegetación húmeda tropical en el llano subandino. Proyecto Amazónica de la Universidad de Turku y Oficina Nacional de Evaluación de Recursos Naturales. Jyväskylä, Finlandia. 265 pp.

MISSOURI BOTANICAL GARDEN - w³ TROPICOS. 2002. Caraipa utilis, C. tereticaulis, Euterpe catinga, Pachira brevipes. URL: http://mobot.mobot.org/cgi-bin/search_vast.

REVILLA, J. 1974. Descripción de los tipos de vegetación en Mishana, Río Nanay. Loreto, Perú. PAHO. Project AMRO-0719 Report. Pan American Health Organization, Washington, D.C.

RUOKOLAINEN, K.; TUOMISTO, H. 1993. La vegetación de terrenos no inundables (tierra firme) en la selva baja de la Amazonía peruana. En: Kalliola, R.; Puhakka, M.; Danjoy, W. (eds.). Amazonía peruana. Vegetación húmeda tropical en el llano subandino. Proyecto Amazónica de la Universidad de Turku y Oficina Nacional de Evaluación de Recursos Naturales. Jyväskylä, Finlandia. 139-153 pp. 
RUOKOLAINEN, K. TUOMISTO, H. 1998. Vegetación Natural de la Zona de Iquitos. En: Kalliola, R.; Flores Paitán, S. (eds.). Geoecología y desarrollo amazónico: estudio integrado en la zona de Iquitos, Perú. Annales Universitatis Turkuensis Ser. A II. Tom. 114. 253-365 pp.

SIAMAZONIA - Sistema de Información de la Diversidad Biológica y Ambiental de la Amazonía Peruana. www.siamazonia.org.pe.

TUOMISTO, H.; RUOKOLAINEN, K. 1994. Distribution of Pteridophyta and Melastomataceae along an edaphic gradient in an Amazonian rain forest. Journal of Vegetation Science 5 (1): 25-34.

VÁSQUEZ, R. 1993. Una nueva Haploclotra (Clusiaceae) de la Amazonía peruana. Novon 3: 499-501.

WALTER, H.; BRECKLE, S.W. 1967. Life Zone Ecological Systems of the Geobiosphere. Tropical and Subtropical zonobiones. Springer Verlag, Berlin. 465 pp. 